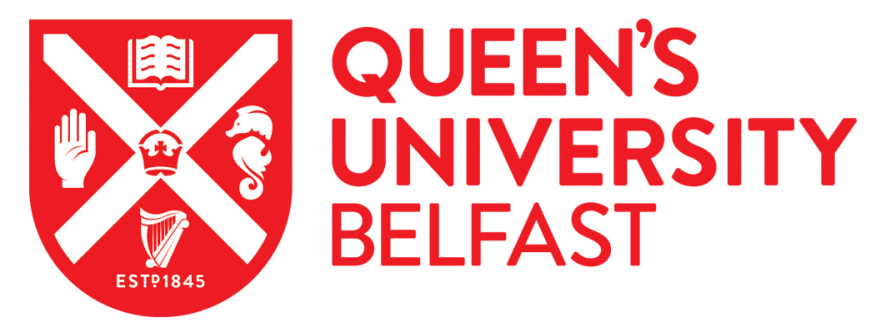

\title{
Epigenetic therapy for the treatment of hypertension-induced cardiac hypertrophy and fibrosis
}

Watson, C. J., Horgan, S., Neary, R., Glezeva, N., Tea, I., Corrigan, N., McDonald, K., Ledwidge, M., \& Baugh, J. (2016). Epigenetic therapy for the treatment of hypertension-induced cardiac hypertrophy and fibrosis. Journal of Cardiovascular Pharmacology and Therapeutics, 21(1), 127-37. https://doi.org/10.1177/1074248415591698

Published in:

Journal of Cardiovascular Pharmacology and Therapeutics

Document Version:

Peer reviewed version

Queen's University Belfast - Research Portal:

Link to publication record in Queen's University Belfast Research Portal

Publisher rights

Copyright 2016 The Authors.

This work is made available online in accordance with the publisher's policies. Please refer to any applicable terms of use of the publisher.

\section{General rights}

Copyright for the publications made accessible via the Queen's University Belfast Research Portal is retained by the author(s) and / or other copyright owners and it is a condition of accessing these publications that users recognise and abide by the legal requirements associated with these rights.

Take down policy

The Research Portal is Queen's institutional repository that provides access to Queen's research output. Every effort has been made to ensure that content in the Research Portal does not infringe any person's rights, or applicable UK laws. If you discover content in the Research Portal that you believe breaches copyright or violates any law, please contact openaccess@qub.ac.uk. 
Epigenetic therapy for the treatment of hypertension induced cardiac hypertrophy and fibrosis

Chris J. Watson ${ }^{a, b, \dagger}$, Stephen Horgann ${ }^{a, \dagger}$, Roisin Neary ${ }^{a}, N^{2}$ adezhda Glezeva ${ }^{a}$, Isaac Tea ${ }^{a}$, Niamh Corrigan ${ }^{a}$, Ken McDonald ${ }^{b}$, Mark Ledwidge ${ }^{b}$, John Baugh ${ }^{a}$.

a School of Medicine \& Medical Science, UCD Conway Institute, University College Dublin, Dublin, Belfield, Dublin 4, Ireland.

b Chronic Cardiovascular Disease Management Unit, St Vincent's Healthcare Group/St Michael's Hospital, Co. Dublin, Ireland.

${ }^{\dagger}$ Equal author contribution

Correspondence to Chris Watson, PhD, School of Medicine \& Medical Science, UCD Conway Institute, University College Dublin, Belfield, Dublin 4, Ireland.

E-mail chris.watson@ucd.ie; Tel. +35317166711; Fax+35317166701.

Acknowledgments: Funding support was provided by University College Dublin Seed Funding Scheme and the Health Research Board of Ireland. 


\section{Abstract}

Background: The development of heart failure is associated with changes in the size, shape, and structure of the heart that has a negative impact on cardiac function. These pathological changes involve excessive extracellular matrix deposition within the myocardial interstitium and myocyte hypertrophy. Alterations in fibroblast phenotype and myocyte activity are associated with reprogramming of gene transcriptional profiles which likely requires epigenetic alterations of chromatin structure. The aim of our work was to investigate the potential of a currently licenced anticancer epigenetic modifier as a treatment option for cardiac diseases associated with hypertensioninduced cardiac hypertrophy and fibrosis.

Methods and Results: The effects of DNA methylation inhibition with 5-azacytidine was examined in a human primary fibroblast cell line and in a spontaneously hypertensive rat model.

The results from this work allude to novel in vivo anti-fibrotic and anti-hypertrophic actions of 5azacytidine. Administration of the DNA methylation inhibitor significantly improved several echocardiographic parameters associated with hypertrophy and diastolic dysfunction. Myocardial collagen levels and myocyte size were reduced in 5-azacytidine-treated spontaneously hypertensive rats. These findings are supported by beneficial in vitro effects in cardiac fibroblasts. Collagen I, collagen III and alpha smooth muscle actin were reduced in a human ventricular cardiac fibroblast cell line treated with 5-azacytidine.

Conclusion: These findings suggest a role for epigenetic modifications in contributing to the profibrotic and hypertrophic changes evident during disease progression. Therapeutic intervention with 5azacytidine demonstrated favourable effects highlighting the potential use of this epigenetic modifier as a treatment option for cardiac pathologies associated with hypertrophy and fibrosis.

Keywords: Hypertension; cardiac fibrosis; hypertrophy; DNA methylation; epigenetics; 5-azacytidine 


\section{Introduction}

Cardiac hypertrophy, characterised by thickening of the myocardium, occurs as an adaptive response to pressure overload, metabolic disarray, mutations of sarcomeric proteins, or loss of contractile mass from prior infarction ${ }^{1}$. Consequently, left ventricle (LV) mass increases whilst chamber size may remain unchanged or decrease (eccentric hypertrophy) or increase (concentric hypertrophy) leading to impaired cardiac function. Left ventricular hypertrophy $(\mathrm{LVH})$ eventually leads to congestive heart failure, a major cause of morbidity and mortality worldwide ${ }^{2,3}$. Development of heart failure (HF) is associated with adverse LV remodelling and is becoming an ever more prevalent condition as risk factors such as hypertension, diabetes, and obesity continue to be poorly controlled ${ }^{4-6}$.

Most drivers of cardiac injury and stress cause an increase in extracellular matrix (ECM) deposition as well as myocyte hypertrophy and it is the combination of these factors that lead to LVH and decreased organ function. Exaggerated ECM deposition or fibrosis is common in cardiac hypertrophy, and it has been suggested that alterations in the cardiac interstitium may contribute to changes in diastolic function of hypertrophied hearts ${ }^{7}$. It has also been suggested that myocardial fibrosis may restrict myofibrillar motion and thereby impair overall cardiac function ${ }^{8}$. Fibrosis is essentially an over-active wound healing response characterised by fibroblast accumulation, proliferation, and activation. In response to tissue injury, fibroblasts differentiate into contractile alpha smooth muscle actin ( $\alpha$-SMA) positive myofibroblasts and synthesise ECM components including collagens and fibronectin. This is an important physiological adaptation that maintains organ structural integrity after injury but when inappropriately controlled exaggerated myofibroblast activation can have a significant impact on the mechanical properties of myocardial tissue. The resultant remodelling of the heart ultimately leads to a decrease in ventricular compliance together with ventricular hypertrophy, conduction abnormalities, increased blood pressure and endothelial dysfunction ${ }^{9}$. 
Physiological adaptive myocyte hypertrophy is likely predominantly driven by mechanical mechanisms. In response to pressure or volume overload, individual cardiac myocytes become mechanically stretched and activate intracellular hypertrophic signalling pathways leading to the activation of embryonic transcription factors and increased synthesis of various structural and contractile proteins. .

It is likely that sustained alterations in fibroblast phenotype and myocyte activity involve reprogramming of gene transcriptional profiles and epigenetic alterations of chromatin structure. Defined as a set of mechanisms that influence gene expression without altering the DNA sequence; epigenetics plays an essential role in the regulation of gene expression. Alterations include DNA methylation, histone modifications and micro-RNA changes.

DNA methylation involves the addition of methyl groups to cytosine residues already incorporated into DNA sequences. The reaction is catalysed by the DNA methyltransferase enzymes (DNMTs) and results in the formation of 5-Methylcytosine (5MeC). This generally causes gene repression either by physically preventing transcription factor binding or reducing access through local chromatin condensation.

Interestingly, the pathogenesis of fibrosis in several other organ systems is associated with gene expression changes involving DNA methylation. In the lung, alterations in DNA methylation patterns have been associated with deregulated expression of a number of genes associated with fibroblast activation and pulmonary fibrosis ${ }^{10-13}$. In addition to this, a role for DNA methylation in liver and renal fibrosis has also been alluded to. Separate studies have identified gene specific hypermethylation events which contribute to fibroblast differentiation ${ }^{14,15}$.

Given the associations highlighted above linking hypermethylation and the development of fibrosis in other organ systems together with the lack of effective therapeutics targeting the cardiac fibrotic response, we hypothesise that epigenetics may play a role in cardiac 
hypertrophy and fibrosis and that intervention with the DNA methylation inhibitor 5azacytidine may have beneficial anti-fibrotic effects. 5-azacytidine (5aza) can bind to and inhibit the actions of DNA methyltransferases, the enzymes responsible for maintaining the methylation pattern of cells, resulting in global DNA hypomethylation and subsequent alterations in gene expression. 5aza and 5-aza-2-deoxycytidine (5-azadC) are currently licensed for the treatment of myelodysplasic syndromes (MDS). This 5-aza/5-azadC treatment of MDS is believed to exert its primary antineoplastic effects by directly incorporating into DNA causing hypomethylation with the resultant re-expression of epigenetically silenced genes. In the heart, drug-induced DNA hypomethylation is likely to affect many genes and this may have a positive effect on cardiac hypertrophy and fibrosis due to the inevitable involvement of networks of proteins and pathways in reprogramming hypertrophy and fibrosis.

Therefore the purpose of this study was to examine the effect of DNA methylation inhibition with 5-aza in a spontaneously hypertensive rat (SHR) model of cardiac hypertrophy and fibrosis and to determine whether there was a potential role for drug repurposing in the treatment of these pathologies.

\section{Methods}

\section{Primary cell culture and treatments}

Primary human ventricular cardiac fibroblast cells (HCF) were purchased from ScienCell Research Laboratories. Cells were cultured and maintained in Dulbecco's modified eagles medium (DMEM) (Gibco), supplemented with 10\% Fetal Bovine Serum (Gibco) and penicillin-streptomycin antibiotics (Gibco) in a $5 \% \mathrm{CO}_{2}$ humidified incubator at $37^{\circ} \mathrm{C}$. When required, HCF cells were treated for up to 8 days with either $5 \mathrm{ng} / \mathrm{ml}$ human recombinant 
transforming growth factor beta 1 (TGF $\beta 1$ ) (R\&D Systems), $5 \mu \mathrm{M}$ 5-azacytidine (5-aza) (Sigma), or with both simultaneously.

Rat cardiac myoblast cells (H9c2) were purchased from American Type Culture Collection (ATCC) and were maintained in low bicarbonate DMEM growth medium under the conditions described above.

In vitro experiments were repeated $n=3$ times.

\section{Quantitative real-time PCR}

RNA isolation from HCF and H9c2 cells was achieved using NucleoSpin RNA II Kit (Macherey-Nagel). Total RNA was also isolated from frozen rat myocardial tissue stored in RNALater Tissue Reagent (Qiagen). RNA extraction was performed using an RNeasy Mini kit according to the manufacturer's instructions (Qiagen). RNA quality and concentration were then determined by spectrophotometry (Nanodrop - Thermo Scientific).

First strand cDNA synthesis was carried out using SuperScript II RT (Invitrogen). Quantitative real-time PCR (QPCR) primers were designed so that one of each primer pair was exon/exon boundary spanning to ensure only mature mRNA was amplified. Primer sequences are as follows; aSMA, 5'-CGTTACTACTGCTGAGCGTGA-3' (forward), 5'AACGTTCATTTCCGATGGTG-3' (reverse); $\quad$ collagen $1 \quad \alpha 1 \quad$ (COL1A1), 5'GAACGCGTGTCATCCCTTGT-3' (forward), 5'-GAACGAGGTAGTCTTTCAGCAACA-3' (reverse); collagen 3 a1 (COL3A1), 5'- AACACGCAAGGCTGTGAGACT-3' (forward), 5'GAACGAGGTAGTCTTTCAGCAACA-3' (reverse). QPCR reactions were normalized by amplifying the same cDNA with beta-2-microglobulin (B2M) primers, 5'AGGCTATCCAGCGTACTCCA-3' (forward), 5'-CCAGTCCTTGCTGAAAGACA-3' (reverse); Myosin binding protein C (MYBP-C3), 5'- CTGGAGACCTGGACCTCAGA-3' (forward), 5'CCGGAAACTGCTCTTCTTCA-3' (reverse). 
QPCR was performed using Platinum SYBR Green qPCR SuperMix-UDG (Invitrogen). Amplification and detection were carried out in duplicate with Mx3000P System (Stratagene). The PCR cycling program consisted of 40 three-step cycles of 15 seconds $/ 95^{\circ} \mathrm{C}, 30$ seconds $/ \mathrm{T}_{\mathrm{A}}$ and 30 seconds $/ 72^{\circ} \mathrm{C}$. To confirm signal specificity, a melting program was carried out after the PCR cycles were completed. Relative fold change in gene expression was calculated with the delta delta CT method.

\section{Western Blotting}

Whole cell protein lysates were generated using RIPA Lysis Buffer (Millipore) with a protease inhibitor cocktail (Roche). Protein concentrations were determined with a BCA Protein Assay Kit (Pierce). Protein lysates were denatured, reduced and resolved on SDSpolyacrylamide gels by SDS-PAGE before transfer onto $0.45 \mu \mathrm{m}$ pore size Immobilon-P polyvinylidene fluoride (PVDF) membranes (Millipore).

Membranes were incubated with blocking buffer (TBS, 0.25\% Tween-20, 0.1\% serum from species that secondary antibody was raised in, and 5 or $10 \%$ fat-free skimmed milk) for 1 hour at room temperature. Membranes were probed overnight with anti-aSMA (Sigma) or anti-Col1A1 (Santa Cruz) antibodies. Detection of the specific binding of the primary antibody was achieved using HRP-conjugated secondary antibodies, followed by signal detection with Immobilon Western chemiluminescent HRP substrate (Millipore). Anti-GAPDH (Millipore) was used to verify equal loading.

\section{5-azacytidine treatment of a rat model of hypertensive heart disease}

Approval from the local Animal Research Ethics Committee was sought and obtained to investigate the impact of 5-aza on cardiac structure, function and fibrosis. All animals used in the study received humane care and the study protocol complied with the institution's guidelines. Male spontaneously hypertensive rats (SHR) and their normotensive 
counterparts - Wistar Kyoto rats (WKY) were purchased from Charles River. From 10 weeks of age the animals received alternate day intra-peritoneal injection of either PBS (vehicle) or 5 -aza $(10 \mathrm{mg} / \mathrm{kg})^{14}$ for 12 weeks. 5-aza (Sigma) was diluted in sterile PBS and filtered through a $0.22 \mu \mathrm{m}$ filter. Aliquots were stored at $-20^{\circ} \mathrm{C}$ and used within 5 days of reconstitution. The study design consisted of three groups of 10 animals; group 1 included 10 SHR rats who received 5-aza (SHR-5-aza); group 2 included 10 SHR rats who received PBS vehicle (SHR-V); group 3 included 10 WKY rats who received PBS vehicle (WKY-V). All rats were housed in an animal facility under identical conditions, with a 12 hour light-dark cycle.

\section{Systolic blood pressure measurements}

Systolic blood pressure was measured using the non-invasive tail-cuff method (Letica Scientific Instruments LE 5001). Blood pressure values were recorded while the animals were under inhaled anaesthesia ( $2 \%$ isoflurane). The mean of three consecutive measurements was obtained for each animal at study mid-point (6 weeks) and end of study (12 weeks).

\section{Doppler Echocardiography}

Cardiac structure and function was assessed at baseline and at the end of the study (12 weeks) using Echocardiography. During the procedure, the animals were under inhaled anaesthesia (isoflurane 2\%) and body temperature was maintained using a heat mat. Echocardiography assessment was performed using a Vevo 770 High-Resolution In Vivo Micro-Imaging System (Visualsonics) with a $10 \mathrm{mHz}$ transducer. M-mode and 2-dimensional (2D) images were obtained in the parasternal long- and short-axis views. The interventricular septal thickness, posterior wall thickness, and LV diameter were measured in systole and diastole at the tips of the papillary muscle. Measurements were taken over three consecutive 
cardiac cycles and averaged. Left ventricular mass (LVM) was calculated according to Devereux's formula and indexed to tibial length (LVMi). Blinded analysis was performed by two separate independent observers. Tissue Doppler images of the lateral mitral valve annulus were acquired and peak E' determined.

\section{Myocardial rat tissue collection and preparation}

On completion of the in vivo rat study, animals were sacrificed (terminal bleed while under inhaled $4 \%$ isoflurane anaesthesia), and the heart was removed en-bloc to study the impact of 5-aza on collagen deposition within the myocardium. Two methodological approaches were used to quantify collagen deposition, staining of cardiac tissue sections using picrosirus red and hydroxyproline assay using tissue lysates.

For picrosirius red staining, the left ventricular mid-sections (papillary level) of the hearts were dissected immediately following sacrifice, rinsed in PBS, and fixed with $10 \%$ formalin (Sigma). Formalin-fixed tissue was embedded in paraffin and $5 \mu \mathrm{m}$ thick tissue sections were created for collagen analysis using picrosirius red.

For hydroxyproline quantification, the left ventricular base of the heart was dissected immediately following sacrifice, rinsed in PBS, and snap-frozen in liquid nitrogen until required for analysis. Frozen hearts were thawed on ice and individually disrupted and homogenized using an Ultra Turrax T25 Dispersing Instrument (IKA). Total protein within tissue lystates were quantified and $10 \mu \mathrm{g}$ of homogenate was used to determine hydroxyproline content.

\section{Hydroxyproline assay}

In brief, $500 \mu$ l of homogenised cardiac tissue sample (ratio of $100 \mathrm{mg}$ tissue homogenised in $1 \mathrm{ml}$ PBS) was incubated at $37^{\circ} \mathrm{C}$ in a vacuum oven overnight in $1 \mathrm{ml} 6 \mathrm{~N} \mathrm{HCl}$. Five 
microlitres of citrate/acetate buffer $(7.24 \%$ sodium acetate, $5 \%$ citric acid, $3.4 \%$ sodium

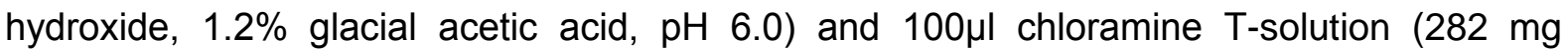
chloramine T, $2 \mathrm{ml} \mathrm{n}$-propanol, $2 \mathrm{ml} \mathrm{H}_{2} \mathrm{O}, 16 \mathrm{ml}$ citrate/acetate buffer) were added to $5 \mu \mathrm{l}$ of the digested cardiac tissue sample, and incubated for 20 minutes at room temperature. Following incubation, $100 \mu \mathrm{l}$ of Ehrlich's solution (2.5g 4-[dimethylamino] benzaldehyde [4DMAB], $9.3 \mathrm{ml} \mathrm{N}$-propanol, $3.9 \mathrm{ml} 70 \%$ perchloric acid) was added to each sample and incubated for 20 minutes at $65^{\circ} \mathrm{C}$. Samples were subsequently cooled for 10 minutes and read at 550nm using a SpectraMax M2 plate reader (Molecular Devices) with SoftMax Pro software (Molecular Devices, version 4.7.1). In parallel, a hydroxyproline standard curve was created to generate quantifiable data. Hydroxyproline (Sigma) concentrations from 0$200 \mu \mathrm{g} / \mathrm{ml}$ were used and were handled in a similar fashion to the digested homogenised cardiac tissue samples.

\section{Picrosirius red staining and automated digital quantification}

Tissue sections were deparaffinised and rehydrated prior to incubating with $0.2 \%$ phosphomolybdic acid (PMA) for 2 minutes. After rinsing in distilled water, the slides were stained with picrosirus red (Direct Red 80 dissolved in picric acid, Sigma) for 90 minutes. Finally, the slides were placed in $0.4 \%$ hydrochloric acid $(\mathrm{HCl})$ for 2 minutes, $70 \%$ ethanol for 45 seconds, dehydrated and cover-slipped for analysis.

The degree of collagen deposition was quantified by automated digital image analysis (Aperio ScanScope XT Slide Scanner (Aperio Technologies))at 20x magnification. Automated image analysis was performed using Imagescope (Aperio). A positive pixel count algorithm was used to automatically quantify the area occupied by the dark pink stain colours representing collagen within each scanned slide image. Calibration of individual staining patterns was performed by specifying the requisite colour (range of hues and saturation) and limits for the desired intensity range. Required input parameters for each stain were based on the HSI (Hue, Saturation and Intensity) colour model. To detect the 
dark pink colour of collagen with picrosirus red, a hue value of 0.8 was specified. The hue width value of 0.5 was used to allow inclusion of a moderate range of colour shades. A perivascular collagen volume fraction was calculated based on the percent of dark pink collagen staining quantified around each blood vessel and adjusted to luminal area ${ }^{16-18}$.

\section{Cardiomyocytes size measurement}

Relative cardiomyocyte size was quantified in haematoxylin and eosin stained mid-LV sections. Cell area was measured in transverse section by planimetry in the presence of a centrally located nucleus. Two independent observers were blinded and three fields of 10 myocytes per slide (30 cells in total per section) were measured using the Aperio digital image analysis system ${ }^{19,20}$.

\section{Statistical analysis}

Comparisons between the control and treatment groups were made using independent t-test or ANOVA (Tukey post-hoc analysis), where appropriate, with $p$ values $<0.05$ considered statistically significant. All statistical calculations were performed using Graph Pad prism Software (Version 4, San Diego, CA).

\section{Results:}

5-aza reduces TGF $\beta 1$-induced Collagen 1, Collagen 3 and Alpha smooth muscle actin mRNA expression in human cardiac fibroblasts

The effect of DNA methylation inhibition on the expression of collagen 1 and 3 was examined in TGF $\beta 1$-stimulated fibroblasts by quantitative real-time PCR. Cardiac fibroblasts were pre-treated with $5 \mu \mathrm{M} 5$-aza for four days prior to stimulation with $5 \mathrm{ng} / \mathrm{ml}$ TGF $\beta 1$. An increase in expression of collagen 1 and 3 was seen in TGF $\beta 1$-stimulated cells. Treatment 
with 5 -aza significantly reduced this increase in collagen $1(p<0.01)$ and collagen 3 $(p<0.001)$, Figure $1 A$ and $B$.

A significant increase in $\alpha$ SMA gene expression in TGF $\beta 1$-treated fibroblasts was seen and there was a trend towards reduction of this marker upon 5-aza treatment, Figure 1C.

\section{5-aza decreases TGF $\beta 1$-induced protein expression of Collagen 1 and Alpha Smooth muscle action in human cardiac fibroblasts}

Collagen 1 and aSMA protein expression was analysed to assess the effect of 5-aza on TGF $\beta 1$-induced fibroblast differentiation and activation (Figure 1D). An increase in both collagen 1 and aSMA expression was observed in stimulated cells. Treatment with 5-aza ameliorated TGF $\beta 1$-induced collagen 1 expression. TGF $\beta 1$ treatment also increased $\alpha$ SMA protein expression and 5-aza significantly reduced its expression (Figure 1D).

\section{Effect of 5-aza on echocardiographic parameters and blood pressure}

Echocardiography was performed at baseline and 12 weeks to evaluate heart structure and function. Ventricular hypertrophy was assessed using M-mode echocardiography (Figure 2A). Interventricular septum diameter (IVSD) and left ventricular mass index (LVMI) were used to assess left ventricular hypertrophy (LVH). IVSD and LVMI were significantly increased in SHR compared to WKY normotensive controls. Therapeutic intervention with 5aza significantly reduced both IVSD and LVMI at 12 weeks, $p<0.01$ (Figure B and C). Diastolic function was assessed in all animals with $E$ prime (E') as a robust echocardiographic measure of diastolic dysfunction. The SHR group displayed a significant reduction in E' compared to normotensive WKY control. E' was significantly improved following 5-aza treatment $(p<0.05)$ and levels were comparable to those of normotensive WKY controls, Figure 2D. Reduced ejection fraction in the SHR group was also significantly

improved with 5-aza treatment (Figure E). Systolic blood pressure (SBP) was assessed at 
12 weeks. Blood pressure was significantly elevated in the SHR group. Administration of 5aza had no effect on the hypertension observed in the SHR, Figure $2 \mathrm{~F}$.

\section{Cardiac tissue analysis}

\section{Effect of 5-aza on myocardial fibrosis}

The impact of 5-aza administration on collagen levels was assessed. Total soluble collagen in cardiac tissue homogenates was determined by hydroxyproline assay. A significant reduction in total collagen was observed in SHR rats treated with 5-aza compared to SHR vehicle controls, $p<0.05$ (Figure 3A).

Histological analysis of collagen was assessed by picrosirius red staining of paraffinembedded sections. Collagen was found to be localised to perivascular areas.

The degree of collagen deposition, reflected by the magnitude of picrosirius red staining, was quantified by automated digital image analysis. A positive pixel count algorithm was used to automatically quantify the area occupied by stain colours within each scanned slide image. Application of the algorithm generated a mark-up image highlighting positive pixels as yellow, orange or red, and negative pixels as blue.

Results from digital analysis highlight that perivascular collagen deposition was significantly reduced in animals treated with 5-aza compared to untreated SHR animals, $p<0.05$ (Figure $3 \mathrm{~B})$. Figure $3 \mathrm{C}$ is a representative image highlighting the creation of a mark-up image following the application of the positive pixel count algorithm with regions of blue representing negative staining pixels, and the yellow, orange, and red pixels represent weak, moderate and strong positive staining collagen respectively.

\section{Effect of 5-aza on cardiac myocytes in vivo}

Myocyte area is directly related to cardiac hypertrophy. To assess the ability of 5-aza to reduce myocyte hypertrophy cell area was measured in H\&E stained cardiac tissue. Quantification using the Aperio digital image analysis system revealed that the average 
myocyte area in SHR 5-aza animals was significantly smaller than that of their counterpart SHR controls, $p<0.001$ (Figure $4 A$ ). This result is consistent with the observed reduction in LVMI observed during echocardiographic analysis.

\section{5-aza up-regulates gene expression of MYBP-C3}

Dysregulation of the cardiac myosin-binding protein C3 (MYBP-C3) gene is linked to cardiac hypertrophy, with up to $40 \%$ of hypertrophic cardiomyopathies exhibiting disease-causing mutations in this gene ${ }^{21}$. Upregulation of MYBP-C3 may have beneficial effects on the myocardium ${ }^{22}$. Inhibiting DNA methylation may also improve cardiac function through upregulation of this gene.

Treatment with 5-aza significantly up-regulated MYBP-C3 gene expression in the SHR group treated with this inhibitor compared to vehicle-treated control animals (Figure 4B). In addition, myoblasts treated with 5-aza in vitro displayed a significant induction of MYBP-C3 gene expression (Figure 4C).

\section{Discussion}

Cardiac hypertrophy and fibrosis are key pathological components of the majority of cardiac diseases that are a result of myocyte hypertrophy and fibrotic collagen deposition. Therapeutic approaches for these pathologies at present target risk factors that drive the disease, and do not directly modify the heart. As a result, novel approaches to treatment are urgently required.

The importance of epigenetics in cardiovascular disease is becoming ever apparent; several studies now allude to associations between epigenetic modifications and the development of cardiac disorders. Previous reports have implicated changes in histone acetylation I deacetylation ${ }^{24-26}$ and differential expression of micro-RNAs ${ }^{27-29}$ in the pathogenesis of cardiac disease. 
Our work has uncovered a role for DNA methylation in maintaining cardiac homeostasis. We show, for the first time, that 5-aza treatment in SHRs has beneficial effects on both myocardial fibrosis and myocyte hypertrophy. This is supported by in vitro experiments in human cardiac fibroblasts where significant protective effects of 5-aza on a pro-fibrotic phenotype are also observed. Administration of the DNA methylation inhibitor significantly reduced gene expression levels of collagen I and collagen III in human ventricular fibroblasts. Protein analysis of collagen 1 and $\alpha$-SMA, a marker of myofibroblast differentiation, also demonstrates attenuating effects of 5-aza on accumulation of these profibrotic markers.

The SHR model is characterised by ventricular hypertrophy, reactive cardiac fibrosis and increased cardiac stiffness ${ }^{30}$. Echocardiographic analysis of cardiac function demonstrates favourable effects of chronic 5-aza administration. Analysing myocardial wall dimensions is useful for assessment of hypertrophy. Therapeutic intervention with 5-aza significantly reduced IVSd and LVMI. Left ventricular mass is one of the most accurate measures of left ventricular hypertrophy in vivo and this is made more sensitive when indexed to body surface area in humans or tibial length in rats ${ }^{31}$. Myocyte hypertrophy was also assessed post-mortem. Inhibiting DNA methylation with 5-aza resulted in reduced average myocyte area thus supporting the results from the echocardiographic analyses. We hypothesise that these improvements in hypertrophy as a result of modulating DNA methylation are likely to reduce the future development of cardiovascular events and improve disease prognosis.

Furthermore, diastolic function was significantly improved. Diastolic dysfunction is the result of myocardial remodelling which can lead to abnormal LV filling, reduced LV compliance, and increased diastolic pressure despite sustained systolic function ${ }^{32}$. This syndrome can eventually lead to heart failure with preserved ejection fraction (HFpEF). Diastolic dysfunction is often associated with myocardial hypertrophy and fibrosis and some reports 
have shown that therapies which reduce ventricular fibrosis can improve diastolic function 33,34. This supports our findings whereby 5-aza prevented a decrease in E', a robust echocardiographic marker of diastolic dysfunction found to be reduced in SHRs. Administration of 5-aza maintained E' at levels equivalent to the normotensive control group. Post-mortem tissue analysis was also undertaken. The impact of 5-aza on fibrosis and collagen deposition was examined ex vivo. Soluble collagen levels, assessed by hydroxyproline assay, were significantly reduced with DNA methylation inhibition. This finding was further supported by tissue staining analysis of perivascular collagen accumulation. Perivascular fibrosis typically precedes interstitial fibrosis and is a key determinant of adverse prognosis that impacts on myocardial oxygen delivery, tissue stiffness, and arrhythmogenic risk ${ }^{35,36}$. Therapeutic intervention with 5-aza significantly decreased perivascular collagen levels compared with SHR vehicle-treated animals.

An interesting finding from this study is the lack of impact of 5-aza on systolic blood pressure. Blood pressure was elevated in SHR vehicle-treated animals and was unchanged in the 5-aza-treated SHR group. Given this, it is reasonable to postulate that the beneficial effects of 5-aza on hypertrophy and fibrosis are a result of modulation at the myocardial tissue level rather than as an indirect effect secondary to blood pressure reduction. Of note, while systolic blood pressure remains unchanged along with a reduction in myocardial mass in the 5-aza treated SHR group, this might have an impact on wall stress. Over time an increase in systolic wall stress could be detrimental on ventricular function. Therefore when applying a therapeutic intervention that reduces myocardial mass in patients with high systolic blood pressure it would be important to ensure anti-hypertensive medications are also administered and optimised to reduce the likelihood of an increase in wall stress occurring. Taken together the above results highlight the importance of this epigenetic alteration in myocyte hypertrophy and myocardial fibrosis and suggest that therapeutic intervention with an epigenetic modifier such as 5-aza may be useful for treating diseases associated with these pathologies. 
Our study builds on a growing body of evidence that suggests a therapeutic role for DNA methylation inhibition for the treatment of cardiovascular disorders.

We have previously investigated the pro-fibrotic impact of hypoxia on cardiac fibroblasts and examined whether alterations in DNA methylation could play a role in this process ${ }^{37}$. Supporting the findings which implicate a role for DNA methylation-induced fibrosis in other organ systems, we highlighted that hypoxia-induced pro-fibrotic changes were associated with global DNA hypermethylation, and increased expression of DNA methyltransferase (DNMT) enzymes in cardiac tissue. Perhaps the most significant finding from this work was the beneficial effect upon the application of the DNA methylation inhibitor 5-azadC that inhibited the pro-fibrotic effects of TGF $\beta$ in cardiac fibroblasts ${ }^{37}$.

Furthermore, a recent study demonstrated increased DNMT3a in activated cardiac fibroblasts and in isoprenaline-treated animals which was associated with RASSAF1A silencing and elevated aSMA expression. Therapeutic intervention with 5-aza restored RASSAF1A expression and reduced expression of pro-fibrotic markers ${ }^{38}$. A mouse model of myocardial infarction and a norepinephrine-induced model of cardiac hypertrophy also alluded to protective effects of inhibiting DNA methylation with 5-aza ${ }^{39,40}$. In the recent study by Xiao et al, the authors report that norepinephrine-induced cardiac hypertrophy was associated with DNA hypermethylation and an increase in cardiac levels of DNA methyltransferases. These changes were associated with alterations in the myocardium proteome, as indicated using a mass-spectrometry approach. 5-aza treatment during the last six days of norepinephrine infusion significantly reduced the associated hypertrophy, global DNA hypermethylation, and corrected the expression pattern of most of the norepinephrineinduced protein alterations in the myocardium.

The implication of epigenetics has been addressed in both heart failure and cardiomyopathies where differential patterns of DNA methylation were identified in patients with these conditions ${ }^{41-43}$. Movassagh et. al. recently uncovered a link between DNA methylation and cardiomyopathies. A genome-wide study of DNA methylation in end-stage 
cardiomyopathy hearts showed that the profile of this epigenetic mark differed significantly from that of healthy hearts within the CpG islands of promoters and within gene bodies ${ }^{43}$.

Our work highlights a novel protective effect of 5-aza in a model of hypertensive heart disease which may be useful for the treatment of hypertrophic cardiomyopathy (HCM). HCM is a disease which commonly arises due to mutations in the sarcomeric genes. It is estimated that 1 in 500 people have $\mathrm{HCM}$, and cardiac myosin binding protein C (MYBP-C3) mutations are reported as one of the most common genetic abnormalities ${ }^{21}$. A study evaluating the methylation status of MYBP-C3 revealed that the gene exhibits methylation sites and therefore exhibits potential to demethylate and increase its expression levels ${ }^{42}$. Re-expressing or increasing the expression levels of MYBP-C3 through gene transfer in vivo has been shown to improve systolic and diastolic contractile function as well as reduce left ventricular wall thickness ${ }^{22}$. Our novel data shows that treatment of SHRs with the DNA methylation inhibitor 5-aza results in a significant increase in MYBP-C3 expression within the myocardium and this coincides with reduced hypertrophy. These effects of 5-aza are confirmed in vitro in cardiac myoblast cells whereby treatment with 5-aza upregulates MYBP-C3 gene expression. Although beyond the scope of this study it would be of value to study the gene specific methylation status of MYBP-C3 in disease models to provide a direct link with demethylation and improved cardiac structure and function.

In summary, although epigenetic alterations have been implicated in the development of many cancers 44,45 , the role of epigenetic changes in myocardial hypertrophy, fibrosis and other cardiac diseases remains relatively underappreciated. Based on our findings, together with the above mentioned evidence linking DNA methylation and cardiac dysfunction, it seems plausible to suggest that DNA methylation plays a role in the pathogenesis of cardiac disease and pharmacological modulation of this process may bring about a beneficial clinical treatment regime. Caution must be noted though regarding the need to further refine the 
dose and treatment regime of 5-aza therapy to minimise potential side effects. As the mechanism by which 5-aza inhibits hypertrophy and fibrosis remains unclear it will be important to identify the affected genes in this process to facilitate the identification of novel mechanism related therapeutic targets.

\section{Acknowledgments}

Funding support was provided by University College Dublin Seed Funding Scheme and the Health Research Board of Ireland.

\section{References}

1. Frey N, Katus HA, Olson EN, Hill JA. Hypertrophy of the heart: a new therapeutic target? Circulation. 2004;109(13):1580-1589.

2. Levy D, Murabito JM, Anderson KM, Christiansen JC, Castelli WP. Echocardiographic left ventricular hypertrophy: clinical characteristics. The Framingham Heart Study. Clinical and experimental hypertension. Part A, Theory and practice. 1992;14(1-2):85-97.

3. Levy D, Garrison RJ, Savage DD, Kannel WB, Castelli WP. Prognostic implications of echocardiographically determined left ventricular mass in the Framingham Heart Study. The New England journal of medicine. 1990;322(22):1561-1566.

4. Prugger $\mathrm{C}$, Keil U, Wellmann J, et al. Blood pressure control and knowledge of target blood pressure in coronary patients across Europe: results from the EUROASPIRE III survey. Journal of hypertension. 2011;29(8):1641-1648.

5. Kenchaiah S, Evans JC, Levy D, et al. Obesity and the risk of heart failure. The New England journal of medicine. 2002;347(5):305-313.

6. Dhingra R, Vasan RS. Diabetes and the risk of heart failure. Heart failure clinics. 2012;8(1):125-133.

7. Weber KT, Pick R, Jalil JE, Janicki JS, Carroll EP. Patterns of myocardial fibrosis. Journal of molecular and cellular cardiology. 1989;21 Suppl 5:121-131.

8. Jalil JE, Doering CW, Janicki JS, Pick R, Shroff SG, Weber KT. Fibrillar collagen and myocardial stiffness in the intact hypertrophied rat left ventricle. Circulation research. 1989;64(6):10411050.

9. Wynn TA. Cellular and molecular mechanisms of fibrosis. The Journal of pathology. 2008;214(2):199-210.

10. Huang SK, Fisher AS, Scruggs AM, et al. Hypermethylation of PTGER2 confers prostaglandin E2 resistance in fibrotic fibroblasts from humans and mice. The American journal of pathology. 2010;177(5):2245-2255. 
11. Sanders YY, Pardo A, Selman M, et al. Thy-1 promoter hypermethylation: a novel epigenetic pathogenic mechanism in pulmonary fibrosis. American journal of respiratory cell and molecular biology. 2008;39(5):610-618.

12. Robinson CM, Neary R, Levendale A, Watson CJ, Baugh JA. Hypoxia-induced dna hypermethylation in human pulmonary fibroblasts is ssociated with thy-1 promoter methylation and the development of a pro-fibrotic Phenotype. Respiratory research. 2012;13(1):74.

13. Sanders YY, Ambalavanan N, Halloran B, et al. Altered DNA Methylation Profile in Idiopathic Pulmonary Fibrosis. Am J Resp Crit Care. 2012;186(6):525-535.

14. Bechtel W, McGoohan S, Zeisberg EM, et al. Methylation determines fibroblast activation and fibrogenesis in the kidney. Nature medicine. 2010;16(5):544-550.

15. Yang JJ, Tao $\mathrm{H}$, Huang $\mathrm{C}$, et al. DNA methylation and MeCP2 regulation of PTCH1 expression during rats hepatic fibrosis. Cell Signal. 2013;25(5):1202-1211.

16. Gao S, Long $\mathrm{CL}$, Wang $\mathrm{RH}$, Wang H. K(ATP) activation prevents progression of cardiac hypertrophy to failure induced by pressure overload via protecting endothelial function. Cardiovascular research. 2009;83(3):444-456.

17. Peng $\mathrm{H}$, Carretero OA, Vuljaj N, et al. Angiotensin-converting enzyme inhibitors: a new mechanism of action. Circulation. 2005;112(16):2436-2445.

18. Lopez B, Gonzalez A, Querejeta R, Larman M, Diez J. Alterations in the pattern of collagen deposition may contribute to the deterioration of systolic function in hypertensive patients with heart failure. Journal of the American College of Cardiology. 2006;48(1):89-96.

19. Xiang W, Kong J, Chen S, et al. Cardiac hypertrophy in vitamin D receptor knockout mice: role of the systemic and cardiac renin-angiotensin systems. American journal of physiology. Endocrinology and metabolism. 2005;288(1):E125-132.

20. He HB, Yang XZ, Shi MQ, Zeng XW, Wu LM, Li LD. Comparison of cardioprotective effects of salvianolic acid $B$ and benazepril on large myocardial infarction in rats. Pharmacological reports : PR. 2008;60(3):369-381.

21. Haas J, Frese KS, Peil B, et al. Atlas of the clinical genetics of human dilated cardiomyopathy. European heart journal. 2014.

22. Merkulov S, Chen X, Chandler MP, Stelzer JE. In vivo cardiac myosin binding protein $\mathrm{C}$ gene transfer rescues myofilament contractile dysfunction in cardiac myosin binding protein $C$ null mice. Circulation. Heart failure. 2012;5(5):635-644.

23. Manabe I, Shindo T, Nagai R. Gene expression in fibroblasts and fibrosis: involvement in cardiac hypertrophy. Circulation research. 2002;91(12):1103-1113.

24. Lee TM, Lin MS, Chang NC. Inhibition of histone deacetylase on ventricular remodeling in infarcted rats. American journal of physiology. Heart and circulatory physiology. 2007;293(2):H968-977.

25. Iyer A, Fenning A, Lim J, et al. Antifibrotic activity of an inhibitor of histone deacetylases in DOCA-salt hypertensive rats. British journal of pharmacology. 2010;159(7):1408-1417.

26. Kao YH, Liou JP, Chung CC, et al. Histone deacetylase inhibition improved cardiac functions with direct antifibrotic activity in heart failure. International journal of cardiology. 2013;168(4):4178-4183.

27. Roncarati R, Anselmi CV, Losi MA, et al. Circulating miR-29a, Among Other Upregulated microRNAs, is the Only Biomarker for Both Hypertrophy and Fibrosis in Patients with Hypertrophic Cardiomyopathy. Journal of the American College of Cardiology. 2013.

28. Dai $\mathrm{Y}, \mathrm{Khaidakov} \mathrm{M}$, Wang $\mathrm{X}$, et al. MicroRNAs involved in the regulation of postischemic cardiac fibrosis. Hypertension. 2013;61(4):751-756.

29. Thum T, Lorenzen JM. Cardiac fibrosis revisited by microRNA therapeutics. Circulation. 2012;126(7):800-802. 
30. Doggrell SA, Brown L. Rat models of hypertension, cardiac hypertrophy and failure. Cardiovascular research. 1998;39(1):89-105.

31. Palmieri V, Dahlof B, DeQuattro V, et al. Reliability of echocardiographic assessment of left ventricular structure and function: the PRESERVE study. Prospective Randomized Study Evaluating Regression of Ventricular Enlargement. Journal of the American College of Cardiology. 1999;34(5):1625-1632.

32. Fan D, Takawale A, Basu R, et al. Differential role of TIMP2 and TIMP3 in cardiac hypertrophy, fibrosis, and diastolic dysfunction. Cardiovascular research. 2014.

33. Brilla CG, Funck RC, Rupp H. Lisinopril-mediated regression of myocardial fibrosis in patients with hypertensive heart disease. Circulation. 2000;102(12):1388-1393.

34. Diez J, Querejeta R, Lopez B, Gonzalez A, Larman M, Martinez Ubago JL. Losartan-dependent regression of myocardial fibrosis is associated with reduction of left ventricular chamber stiffness in hypertensive patients. Circulation. 2002;105(21):2512-2517.

35. Kuwahara F, Kai H, Tokuda K, et al. Hypertensive myocardial fibrosis and diastolic dysfunction: another model of inflammation? Hypertension. 2004;43(4):739-745.

36. Melendez GC, McLarty JL, Levick SP, Du Y, Janicki JS, Brower GL. Interleukin 6 mediates myocardial fibrosis, concentric hypertrophy, and diastolic dysfunction in rats. Hypertension. 2010;56(2):225-231.

37. Watson CJ, Collier P, Tea I, et al. Hypoxia-induced epigenetic modifications are associated with cardiac tissue fibrosis and the development of a myofibroblast-like phenotype. Human molecular genetics. 2013.

38. Tao H, Yang JJ, Chen ZW, et al. DNMT3A silencing RASSF1A promotes cardiac fibrosis through upregulation of ERK1/2. Toxicology. 2014;323C:42-50.

39. Xiao D, Dasgupta $C$, Chen $M$, et al. Inhibition of DNA methylation reverses norepinephrineinduced cardiac hypertrophy in rats. Cardiovascular research. 2014;101(3):373-382.

40. Kim YS, Kang WS, Kwon JS, et al. Protective role of 5-azacytidine on myocardial infarction is associated with modulation of macrophage phenotype and inhibition of fibrosis. Journal of cellular and molecular medicine. 2014.

41. Movassagh M, Choy MK, Goddard M, Bennett MR, Down TA, Foo RS. Differential DNA methylation correlates with differential expression of angiogenic factors in human heart failure. PloS one. 2010;5(1):e8564.

42. Meurs KM, Kuan M. Differential methylation of $\mathrm{CpG}$ sites in two isoforms of myosin binding protein $\mathrm{C}$, an important hypertrophic cardiomyopathy gene. Environmental and molecular mutagenesis. 2011;52(2):161-164.

43. Movassagh M, Choy MK, Knowles DA, et al. Distinct epigenomic features in end-stage failing human hearts. Circulation. 2011;124(22):2411-2422.

44. Esteller M. Epigenetic gene silencing in cancer: the DNA hypermethylome. Human molecular genetics. 2007;16 Spec No 1:R50-59.

45. Esteller M. Epigenetics in cancer. The New England journal of medicine. 2008;358(11):11481159.

\section{Figure Legends:}


Figure 1: Inhibiting cardiac fibroblast DNA methylation reduces the gene and protein expression of pro-fibrotic markers.

Expression of collagen 1 (COL1A1), collagen 3 (COL3A1), and alpha smooth muscle actin ( $\alpha \mathrm{SMA})$ genes (A, B, C) and collagen 1 and $\alpha$ SMA protein (D) in human cardiac fibroblasts treated with $+/$ $5 \mu \mathrm{M}$ 5-azacytidine (5-aza)and +/- 5ng/ml TGF 31 . Bars represent fold expression changes to untreated control. Data represent mean \pm SEM. ${ }^{* \star} p<0.01,{ }^{\star *} p<<0.001$.

Figure 2: Effects of 5-azacytidine treatment on echocardiographic parameters in an in vivo rat model of hypertensive heart disease.

Impact of 12 week treatment of spontaneously hypertensive rats (SHR) with $10 \mathrm{mg} / \mathrm{kg} 5$-azacytidine (5-aza) (3 doses per week) (SHR-5AZA). Normotensive Wistar Kyoto (WKY) rats and PBS vehicletreated SHR (SHR-V) were used as control groups. Ten animals were used in each of the 3 study groups. (A) Representative M-mode echocardiographic images demonstrating different degrees of wall thickness between the 3 study groups at 12 weeks. (B-F) Beneficial effects of therapeutic treatment of SHR with 5-aza on cardiac hypertrophy: interventricular septal diameter (IVSd) (B) and left ventricular mass index (LVMi) (C); diastolic function: E' (D); ejection fraction (EF) (E), and systolic blood pressure (F). Data represent average of two blinded observers and graphed as mean \pm SEM. * $p<0.05,{ }^{* \star} p<0.01,{ }^{* * *} p<0.001$.

\section{Figure 3: Effect of 5-azacytidine treatment on myocardial collagen deposition}

Assessment of total soluble myocardial collagen (hydroxyproline assay) and perivascular collagen (tissue picrosirius red staining) within the myocardium of SHR-V and SHR-5aza animals (A and B respectively(C) An example of a low magnification sample image ( $\mathrm{x} 5$ magnification) of picrosirius red stained myocardial collagen deposition (pink staining). Both interstitial and perivascular collagen staining is apparent. A mark-up image is presented which was generated following the application of the positive pixel count algorithm. Regions of blue represent negative staining pixels. Yellow, orange, and red pixels represent weak, moderate and strong positive staining collagen respectively. Data represent mean \pm SEM. ${ }^{*} \mathrm{p}<0.05$. 
Figure 4: Effect of 5-azacytidine treatment on myocyte area in vivo and in vitro

(A) Effect of 5-azacytidine (5-aza) treatment of SHR animals on cardiac myocyte area obtained by digital analysis of myocyte area in haematoxylin and eosin stained cardiac tissue sections. compared to the Vehicle-treated SHR animals were used as a control group. (B) Gene expression changes of myosin binding protein C3 (MYBP-C3) in SHR-5aza versus SHR-V control animals.(C) MYBP-C3 gene expression changes in cardiac myoblast cells treated in vitro with 5-aza (4 days, $5 \mu \mathrm{M}$ ) Bars represent fold expression changes to untreated control. Data represent mean $\pm S E M$. ${ }^{\star \star} p<0.01$, ${ }^{\star * \star} \mathrm{p}<0.001$. 


\section{Figures:}

Figure 1

A

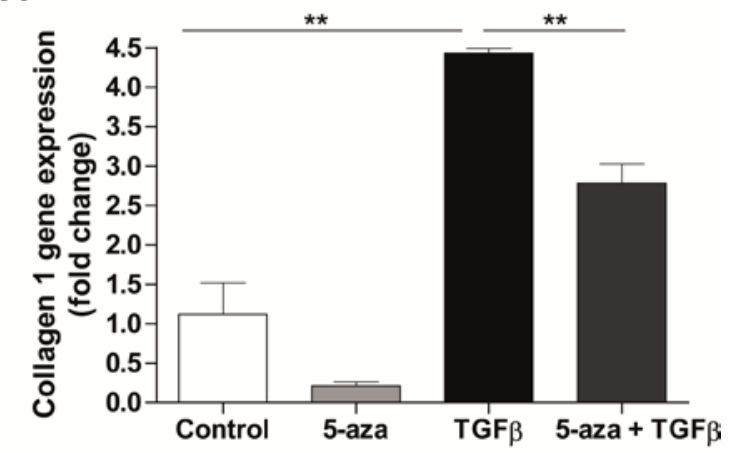

C

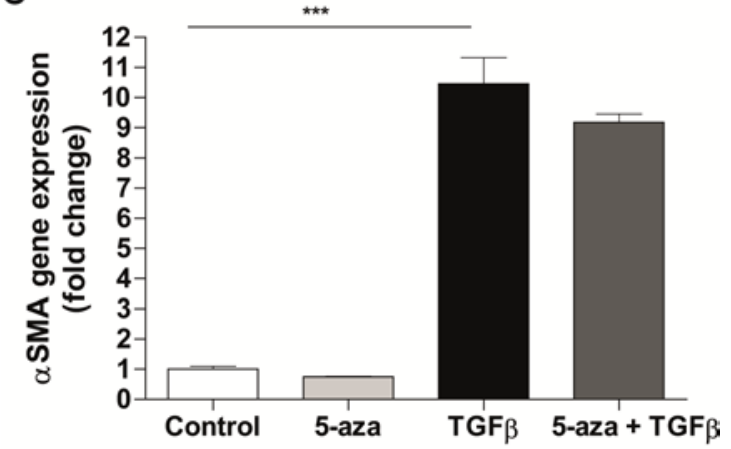

B

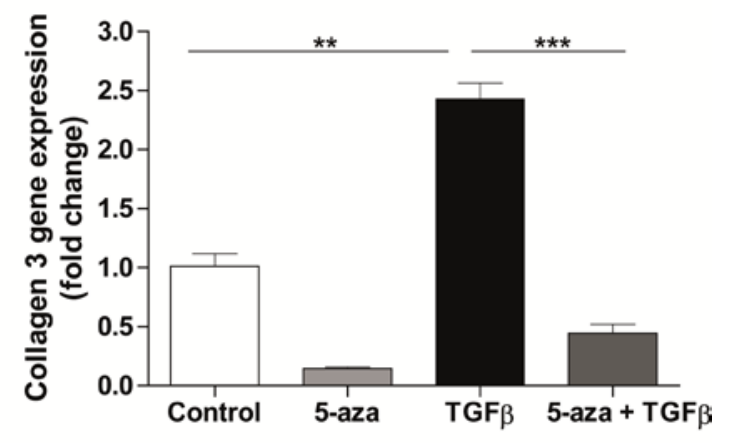

D

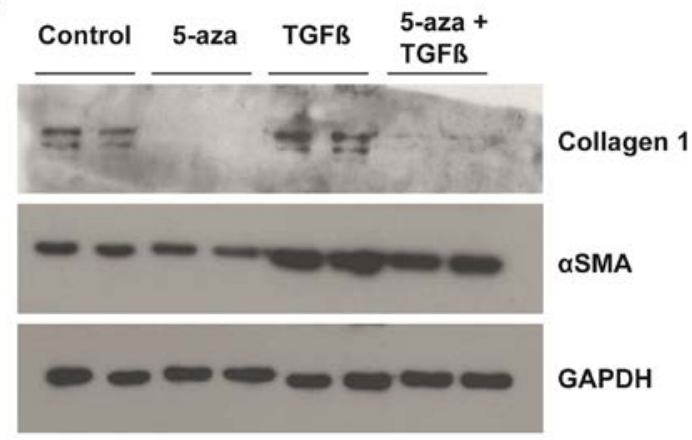


Figure 2

A

WKY

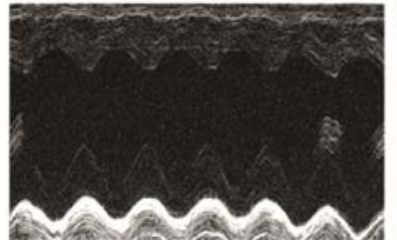

B

Inter-ventricular septum diameter

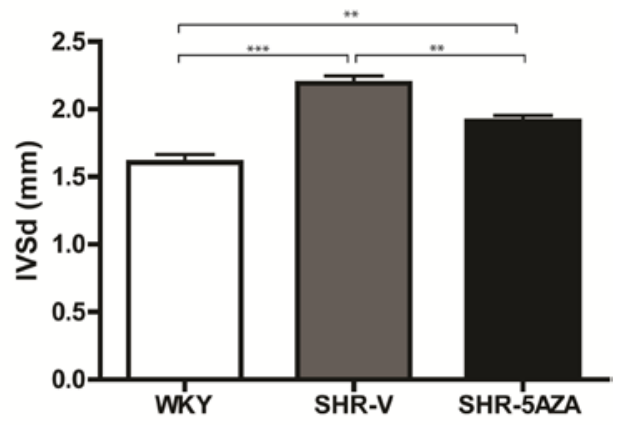

D

E prime

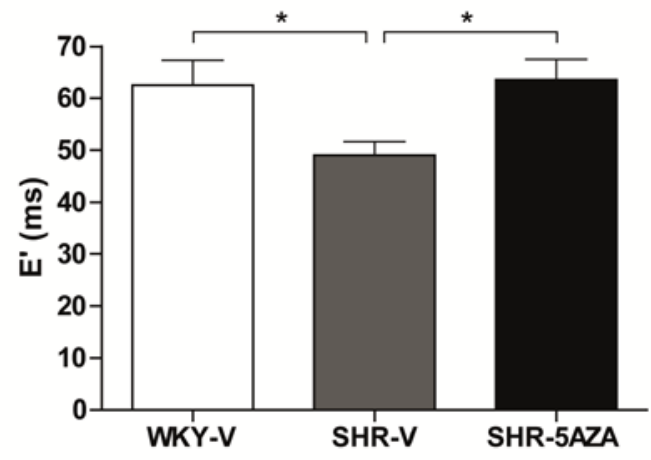

$\mathbf{F}$

Systolic blood pressure

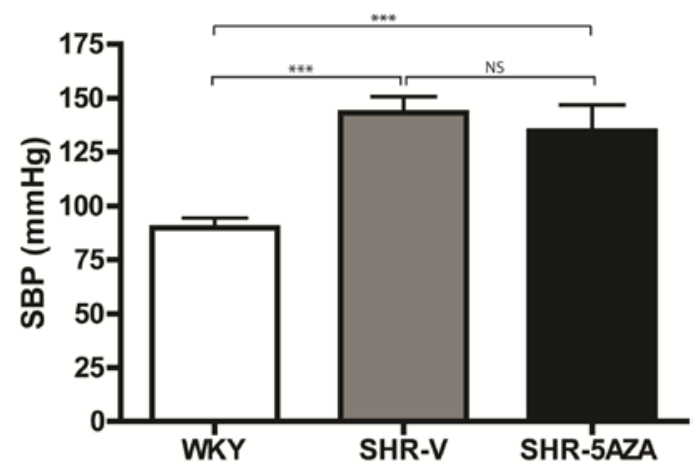

C

Left Ventricular Mass (indexed)

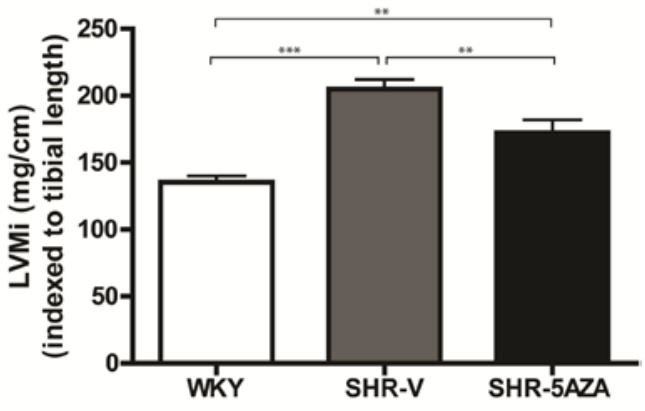

E

Ejection fraction

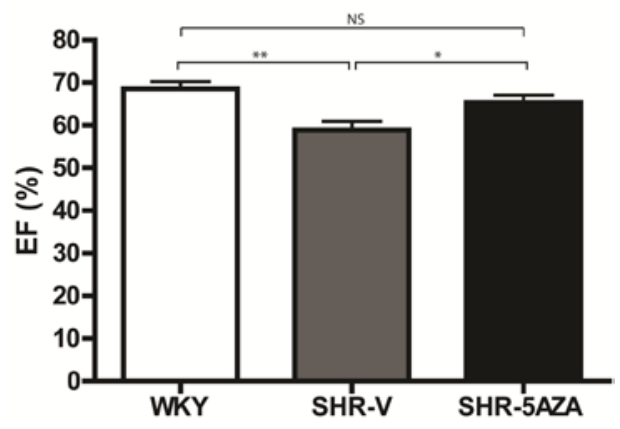


Figure 3

A

Total soluble myocardial collagen

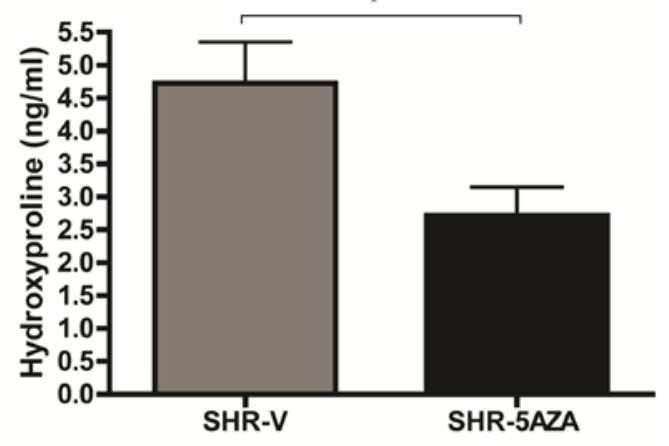

C
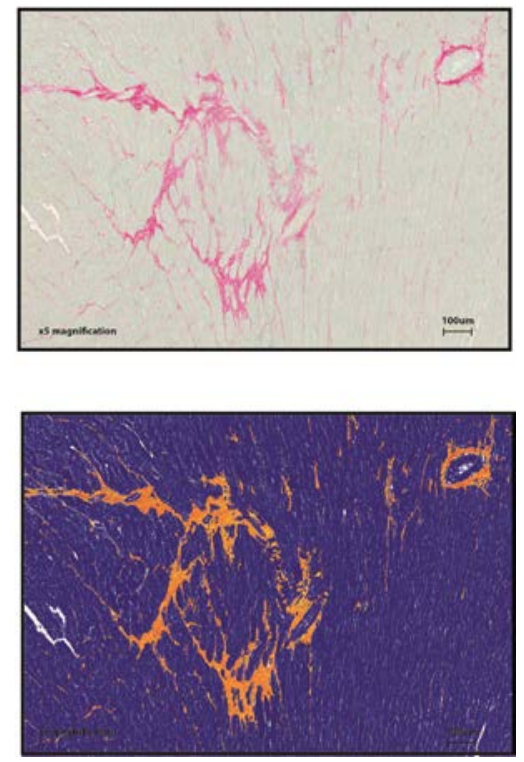

B

Perivascular collagen volume fraction

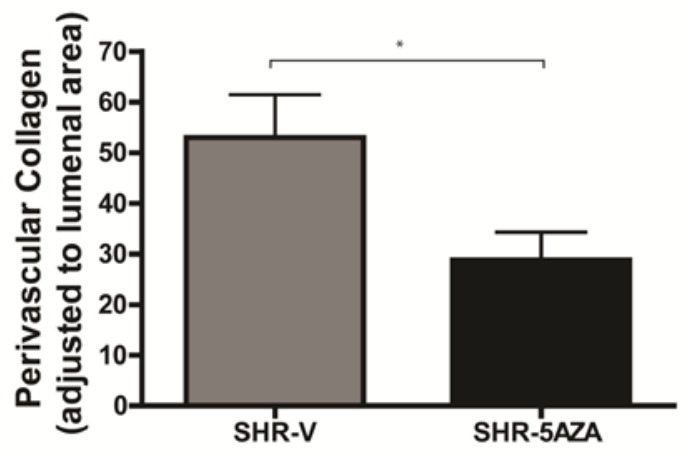


Figure 4

A

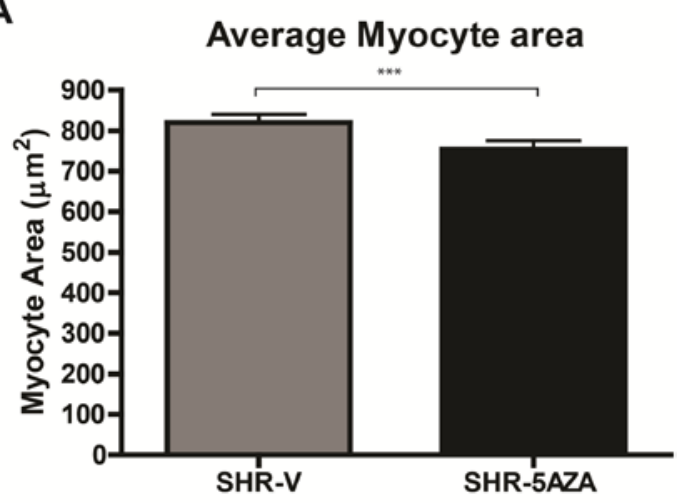

C

Cardiac Myoblasts MYBP-C3

gene expression post 5aza treatment

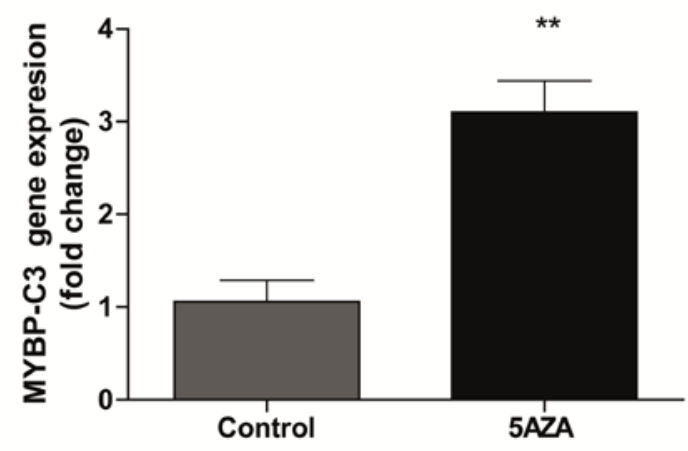

B Myocardial MYBP-C3 gene expression

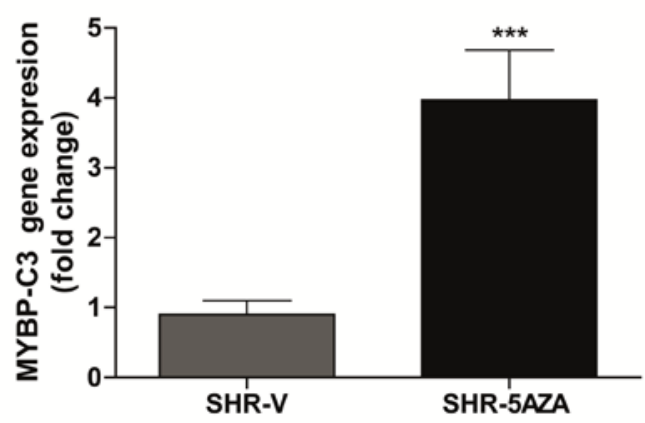


